\title{
The issue of time inconsistency revisited as an extended game*
}

\author{
Roberto Cellini ${ }^{\S}$ and Luca Lambertini\# \\ $\S$ Department of Economics and Quantitative Methods, University of Catania \\ Corso Italia 55, 95129 Catania, Italy \\ phone 39-095375344, fax 39-095-370574, \\ cellini@unict.it \\ \# Department of Economics, University of Bologna \\ Strada Maggiore 45, 40125 Bologna, Italy \\ phone 39-051-2092600, fax 39-051-2092664, \\ lamberti@spbo.unibo.it
}

October 24, 2003

\begin{abstract}
We reformulate the monetary policy model of Barro and Gordon (1983a) by using an extended game with observable delay where the hierarchy of play between the central bank and the private sector is endogenous. This allows us to endogenise the institutional setup wherein the monetary policy game takes place. We show that positive inflation may be observed due to mixed strategies rather than time inconsistency.
\end{abstract}

Keywords: monetary policy, time consistency, extended games

JEL Classification: E52, E61

${ }^{*}$ We thank Matteo Cervellati, Paolo Manasse, Raimondello Orsini and Riccardo Rovelli for insightful discussion. The usual disclaimer applies. 


\section{Introduction}

A classic issue in the existing literature on macroeconomic policy is the time (in)consistency of optimal monetary policy, and the related debate about rules vs discretion. This discussion dates back to Kydland and Prescott (1977). Broadly speaking, the idea behind their analysis and the subsequent literature is that a central bank may strategically exploit an advantage in the game against the private sector (consumers and producers), manoeuvring the inflation rate (through an appropriate choice of the money supply or the nominal interest rate) in order to achieve a higher level of employment and ultimately increase aggregate output. To this aim, the bank finds it convenient to announce that she will manage monetary policy so as to stabilise prices, and then - provided the private sector takes this announcement at face value and adjust inflationary expectations accordingly - she finds it optimal to produce a positive inflation rate (the so-called 'surprise') driving the economy to the desired levels of employment and output. A relevant aspect of the formulation of the problem as it is formulated in Kydland and Prescott (1977) and Barro and Gordon (1983a,b) is the fact that the private sector is not, in fact, a strategic player, but rather an atomistic agent endowed with perfect foresight. This point, which is not outlined in detail in the original papers, is instead discussed in detail by Drazen (2000; see also Rogoff, 1987).

This view on the objectives and behaviour of a central bank originated a debate on how to build a credibility for a central bank, and the desirability of commitment devices limiting her discretion. Eventually, this materialised into the need of mandatory tasks for central bankers, supposed to confine themselves to stabilise prices without trying to affect the demand side of economic systems. Under uncertainty, or incomplete information, announcements and the credibility of monetary policy may rely upon reputational effects generated by a repeated game. ${ }^{1}$

The discussion on the central bank's incentives has also considered a properly dynamic perspective, the first contribution in this vein being that of Calvo (1978). This generated a wide literature, including Lucas and Stokey (1983), Persson et al. (1987), Cohen and Michel (1988), Ireland (1997), Alvarez et al. (2003), inter alia. ${ }^{2}$

\footnotetext{
${ }^{1}$ For exhaustive overviews of the related literature, see Persson and Tabellini (1990, 1999) and Blinder (2000), inter alia.

${ }^{2}$ In particular, this stream of contributions relies upon the idea that the open-loop Stackelberg solution of dynamic games is generally time-inconsistent. To this regard, see
} 
By discussing the alternative between commitment and discretional policies, and the related issue of credibility, all of the aforementioned contributions point to the need of an optimal design of the institutional setup wherein the central bank and the private sector carry out their respective moves. Here, we propose a reformulation of the model by Barro and Gordon (1983a,b), nesting it into an extended game à la Hamilton and Slutsky (1990), ${ }^{3}$ where players (the central bank and the private sector) can endogenously and noncooperatively determine the timing of moves. That is, we propose a way of endogenously modelling the institutional setting representing the backgroung to the model of Kydland and Prescott (1977) and Barro and Gordon (1983a,b). We prove the existence of multiple equilibria: two in pure strategies, one of which is in weakly dominant strategies and entails stable prices, and one in mixed strategies, where a positive inflation rate is observed with positive probability. ${ }^{4}$

Then, we extend the model by including an inflation-aversion term in the preferences of the private sector. This drastically modifies the game, to such an extent that no dominant strategies emerge any more, both for the central bank and for the private sector. The setup becomes a chicken game where both Stackelberg equilibria are subgame perfect equilibrium outcomes. While the one with the bank leading is characterised by stable prices and the output is at the natural level, the other, with the private sector leading, is characterised by a positive inflation rate, so that income is above its natural level. The latter case highlights an issue regarding the way one may think of the private sector. That is, is the private sector summarised by a representative (strategic) agent who knows that his behaviour will significantly interact with the central bank's, or by an atomistic (non strategic) agent, whose only concern is to set expectations rationally, taking the view that he cannot influence the central bank's decision to any appreciable extent? This dual nature of the private sector, already stressed by Drazen (2000), inter alia, clearly reappears here. When the private agent is required to behave strategically, he turns out to have incorrect foresights on the monetary policy of the bank. Conversely, if he is taken to have perfect foresight, then such expectations turn out to be unable to maximise his utility function. The selection between these two equilibria becomes then a matter of coordina-

\footnotetext{
Simaan and Cruz (1973a,b), Kydland (1977) and Xie (1997).

${ }^{3}$ See also d'Aspremont and Gérard-Varet (1980).

${ }^{4}$ The same results obtain if one adopts the model used by Kydland and Prescott (1977).
} 
tion devices and institutional arrangements as well as a matter of assessing whether the private sector is made up by either strategic players or atomistic agents with perfect foresight.

In either case, the bottom line of our analysis is that the occurrence of a positive inflation rate may arise from the strategic interaction between central bank and private agents concerning the timing of moves. In addition to this, it is also worth emphasising that, when the private sector exhibits even the slightest aversion to inflation, then the public itself is better off not forecasting correctly the behaviour of the central bank.

The structure of the paper is as follows. Section 2 presents the basic setup, and reports the solution of the Barro-Gordon model, according to whether the game is played à la Nash or à la Stackelberg. Section 3 presents an extended version of the model, where the timing of moves derives from the players' choices. Section 4 modifies the model taking into account the aversion of private agents toward inflation. Section 5 concludes.

\section{The setup}

The basic model is the same as in Barro and Gordon (1983a,b). The structure of the economy is summarised by an expectation-augmented Phillips curve:

$$
y=\bar{y}+\beta\left(\pi-\pi^{e}\right),
$$

where $y$ is the output level, $\bar{y}$ is the full employment output, $\pi$ is the current inflation rate, $\pi^{e}$ is the expected inflation rate and $\beta$ is a positive parameter measuring the sensitivity of output to the inflationary surprise. We assume perfect foresight on the part of the private sector.

The task of the central bank consists in choosing the inflation rate $\pi$ so as to minimise the following quadratic loss function:

$$
L=\alpha(\pi-\bar{\pi})^{2}+(y-k \bar{y})^{2}, \alpha>0 ; k \geq 1,
$$

under the constraint given by the Phillips curve. Parameter $\bar{\pi}$ defines the supply-side target in therms of a desired inflation rate. In the remainder, we set $\bar{\pi}=0$ for the sake of simplicity. Plugging (1) into (2), the central bank's loss function becomes:

$$
L=\alpha \pi^{2}+\left[\beta\left(\pi-\pi^{e}\right)+(1-k) \bar{y}\right]^{2} .
$$


The private sector sets expectations $\pi^{e}$ so as to minimise the inflationary surprise:

$$
V=\left(\pi-\pi^{e}\right)^{2}
$$

\subsection{The Nash game}

Suppose the central bank and the private sector move simultaneously. The first order conditions (FOCs) are:

$$
\begin{aligned}
& \frac{\partial L}{\partial \pi}=2\left[\alpha \pi+\beta\left(\beta\left(\pi-\pi^{e}\right)+(1-k) \bar{y}\right)\right]=0 \\
& \frac{\partial V}{\partial \pi^{e}}=-2\left(\pi-\pi^{e}\right)=0 .
\end{aligned}
$$

Solving the system (5-6) yields:

$$
\pi_{N}=\frac{\beta(k-1) \bar{y}}{\alpha}=\pi_{N}^{e} ; y_{N}=\bar{y}
$$

with payoffs:

$$
L_{N}=\frac{\left(\alpha+\beta^{2}\right)(k-1)^{2} \bar{y}^{2}}{\alpha} ; V_{N}=0
$$

Subscript $N$ stands for Nash. The Nash equilibrium outcome characterised by expressions (7-8) coincides with the discretional (or third-best) equilibrium in Barro and Gordon (1983a,b).

Barro and Gordon (1983a,b) define as the first-best outcome (for the central bank) the case in which (i) the central bank announces that she will stabilise prices, i.e., $\pi=0$; (ii) the private sector takes the announcement at face value and sets $\pi^{e}=0$; (iii) the central bank minimises $L$ by choosing

$$
\pi=\frac{\beta(k-1) \bar{y}}{\alpha+\beta^{2}}>0
$$

thereby attaining an output level higher than $\bar{y}$. This is seen as the source of the time inconsistency of optimal monetary policy, but it is clearly not an equilibrium outcome as $\pi^{e} \neq \pi$. Therefore, it cannot be part of a subgame perfect equilibrium.

The case where (a) the central bank announces that she will stabilise price, i.e., $\pi=0$; (b) the private sector accordingly sets $\pi^{e}=0$; (c) the central bank sticks to her announcement, so that the outcome $\pi=\pi^{e}=0$ 
obtains, is defined by Barro and Gordon as the second best or commitment equilibrium. Yet, comparing this outcome with the first best, they claim that there exists an ex post incentive to deviate on the part of the bank along her best reply function, yielding the positive inflation rate in (9). In this perspective, only the existence of a pre-commitment technology would allow the central bank to stick to her announcement and achieve price stability. We are about to show that this may not be the case, as price (in)stability is not a matter of announcements and their credibility, but rather a consequence of hierarchical play and selection among multiple pure strategy equilibria.

\section{$2.2 \quad$ The Stackelberg games}

To begin with, consider the case where the private sector is the leader, and solves the following program:

$$
\begin{gathered}
\min _{\pi^{e}} V=\left(\pi-\pi^{e}\right)^{2} \\
\text { s.t. : } \frac{\partial L}{\partial \pi}=2\left[\alpha \pi+\beta\left(\beta\left(\pi-\pi^{e}\right)+(1-k) \bar{y}\right)\right]=0 .
\end{gathered}
$$

It can be immediately verified that the Stackelberg game with the private sector leading is observationally equivalent to the Nash game, i.e., it has the same equilibrium outcome identified by (7-8). That is, given its objective function and the resulting best reply, the private sector cannot gain anything from the possibility of strategically taking into account the central bank's best reply function. However, in such a case inflation is positive:

$$
\pi_{S}=\frac{\beta(k-1) \bar{y}}{\alpha}=\pi_{F}^{e}
$$

Subscripts $F$ and $S$ stands for first and second, respectively. ${ }^{5}$

Now we proceed to examine the Stackelberg game with the central bank leading. Her optimum problem is the following:

$$
\min _{\pi} L=\alpha \pi^{2}+\left[\beta\left(\pi-\pi^{e}\right)+(1-k) \bar{y}\right]^{2}
$$

\footnotetext{
${ }^{5}$ It is worth stressing that this labelling style does not refer to a sequence of moves taking place in calendar time, which is absent here. Rather, it is meant to illustrate a logical timing, i.e., the strategic use of the information contained in the rival's best reply function.
} 


$$
\text { s.t. : } \frac{\partial V}{\partial \pi^{e}}=-2\left(\pi-\pi^{e}\right)=0 \text {, }
$$

yielding:

$$
\begin{gathered}
\pi_{F}=0=\pi_{S}^{e} ; y_{F}=\bar{y} \\
L_{F}=(k-1)^{2} \bar{y}^{2} ; V_{S}=0,
\end{gathered}
$$

with $L_{F}<L_{N}$. The Stackelberg outcome in (13-14) corresponds to the precommitment (or second-best) situation in Barro and Gordon (1983a,b).

\section{The extended game with observable delay}

In order to examine the issue of time consistency of monetary policy, we adopt the toolbox of Hamilton and Slutsky (1990), i.e., we examine an extended game with observable delay where the stage devoted to the choice of $\pi$ and $\pi^{e}$ is preceded by a meta-stage where players choose simultaneously and noncooperatively the timing of moves, that is, whether to move as the first $(F)$ or as the second $(S)$ player. If both the central bank and the private sector choose $F$ (or $S$ ), then they will play simultaneously at the second stage. Otherwise, if one player chooses $F$ and the other chooses $S$, then sequential play is selected. The meta-stage takes place in logical rather than calendar time; accordingly, there is no time discounting. In the light of the discussion summarised in the introduction, the endogenous choice of timing is a way of modelling the optimal design of the institutional setup, prior to the monetary policy game itself. The outcome of the first stage of the extended game, that is, the meta-stage of the game, determines whether the second stage of the game, i.e., the game in the space $\left(\pi, \pi^{e}\right)$ is going to be played à la Nash or à la Stackelberg. The solution of the extended game is thus a subgame perfect equilibrium in two stages. Accordingly, no time inconsistency issue may arise here. The reduced form of the meta-stage is in Matrix 1, where the row player is the central bank $(C B)$ and the column player is the private sector $(P S)$.

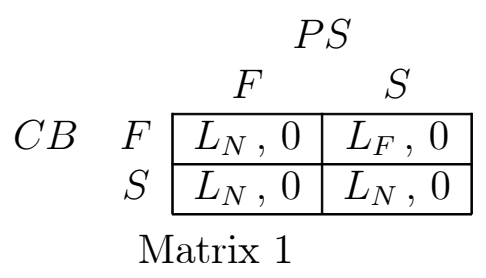


It is easy to verify that the meta-stage produces two pure-strategy Nash equilibria, $(S, F)$ and $(F, S)$, the latter being in weakly dominant strategies. Hence, simultaneous play is ruled out (at least in pure strategies) and along the pure strategy subgame perfect equilibrium path we must expect to observe sequential play with either player leading.

The explanation of this result lies in the fact that the game between the central bank and the private sector is a supermodular one, i.e., the inflation rate and the inflationary expectations are strategic complements. Put it in different but equivalent terms, the best replies that obtain from FOCs (5-6) are everywhere increasing in the space $\left(\pi, \pi^{e}\right)$. Therefore, it must be true that both players are at least as well off in either Stackelberg outcome as in the Nash one. ${ }^{6}$

Moreover, there also exists a mixed strategy equilibrium that can be calculated as follows. Suppose the central bank selects strategy $F$ with probability $q \in[0,1]$ and strategy $S$ with probability $1-q$, while the private sector attaches probability $p \in[0,1]$ to $F$ and $1-p$ to $S$. If so, then the central bank is indifferent between $F$ and $S$ if:

$$
\begin{gathered}
p L_{N}+(1-p) L_{F}=p L_{N}+(1-p) L_{N} \\
\Rightarrow(1-p) L_{N}=(1-p) L_{F}
\end{gathered}
$$

which can be satisfied only at $p=1$, given that $L_{N} \neq L_{F}$. Through the same procedure, it can be ascertained that $q$ is indeterminate, as the private sector's loss function is everywhere nil. Therefore, $(q, 1)$ defines the Nash equilibrium in mixed strategies, for any $q \in[0,1]$.

Summing up, in pure strategies one should expect the emergence of the equilibrium in weakly dominant strategies $(F, S)$ characterised by stable prices $(\pi=0)$. However, with probability $q$, the equilibrium with positive inflation may obtain.

In other words, a positive inflation rate may occur not because of the myopic choice of central bank that is unable to commit herself to a second-best zero-inflation policy (as in the Barro-Gordon story) but because of the existence of multiple equilibria and an associated equilibrium in mixed strategies, in which a positive inflation rate is entailed by the possible outcome where the central bank acts as either a Nash player or a Stackelberg follower.

\footnotetext{
${ }^{6}$ For more on this aspect, see d'Aspremont and Gérard-Varet (1980) and Hamilton and Slutsky (1990).
} 


\section{Extension: inflation-averse private sector}

In this section, we modify the loss function of the private sector to account for the possibility that investors/consumers dislike inflation per se, even if, being endowed with perfect foresight, they can fully anticipate any inflationary manoeuvre by the central bank. Accordingly, we redefine $V$ as follows:

$$
V=\left(\pi-\pi^{e}\right)^{2}+\gamma \pi^{2}
$$

where parameter $\gamma>0$ measures the the aversion to inflation by the private sector. For reasons that will become clear below, we drop the assumption of perfect foresight, or equivalently, we explicitly admit the possibility that the inflation may not be correctly forecasted by the private sector.

In the Nash game, it is easily checked that FOCs (5-6) still hold. However, due to the presence of inflation aversion, now the Nash equilibrium is:

$$
\pi_{N}=\frac{\beta(k-1) \bar{y}}{\alpha}=\pi_{N}^{e} ; y_{N}=\bar{y} .
$$

and the associated losses are:

$$
L_{N}=\frac{\left(\alpha+\beta^{2}\right)(k-1)^{2} \bar{y}^{2}}{\alpha} ; V_{N}=\frac{\beta^{2} \gamma(k-1)^{2} \bar{y}^{2}}{\alpha^{2}},
$$

where $L_{N}$ is obviously the same as in the original Barro-Gordon formulation of the model. The associated inflation rate and output level are also unmodified.

Next, we examine the game where the private sector strategically exploits the information conveyed by the central bank's FOC, or best reply function:

$$
\begin{gathered}
\min _{\pi^{e}} V=\left(\pi-\pi^{e}\right)^{2}+\gamma \pi^{2} \\
\text { s.t. : } \frac{\partial L}{\partial \pi}=2\left[\alpha \pi+\beta\left(\beta\left(\pi-\pi^{e}\right)+(1-k) \bar{y}\right)\right]=0 .
\end{gathered}
$$

The FOC of the leader is:

$$
\frac{\partial V}{\partial \pi^{e}}=\frac{2\left[\alpha^{2} \pi^{e}-\alpha \beta(k-1) \bar{y}+\beta^{2} \gamma\left(\beta \pi^{e}+(k-1) \bar{y}\right)\right]}{\left(\alpha+\beta^{2}\right)^{2}}=0
$$

yielding:

$$
\pi_{F}^{e}=\frac{\beta\left(\alpha-\beta^{2} \gamma\right)(k-1) \bar{y}}{\alpha^{2}+\beta^{4} \gamma}>0 \text { for all } \gamma<\frac{\alpha}{\beta^{2}} .
$$


However, in this case expectations turn out to be incorrect, as the equilibrium inflation rate is:

$$
\pi_{S}=\frac{\alpha \beta(k-1) \bar{y}}{\alpha^{2}+\beta^{4} \gamma}>\pi_{F}^{e}
$$

This finding uncovers a fundamental problem regarding the twofold role of $\pi^{e}$, which is treated as a foresight operator as well as a choice variable at the same time. This, in turn, reveals the conflictual nature of the private sector, which in this case, unlike all the previous ones, cannot be both rational and strategic at the same time (see Drazen, 2000). The result that $\pi_{S}>\pi^{e}$ is due to the supermodularity of the game, that is, to the fact that the reaction functions of both players are everywhere increasing in the choice variable of the opponent. When the public anticipates the behaviour of the central bank and explicitly includes it as a constraint into its optimum problem, then it ends up by expecting unstable prices. Once expectations are fixed, and given the inflation-aversion of the private sector, the central bank reacts by inflating the price level even more than expected. That is, this particular Stackelberg game qualitatively (if not quantitatively) reproduces as an equilibrium outcome what Barro and Gordon (1983a,b) define as the first-best outcome. The difference lies in the fact that in Barro and Gordon the firstbest is relies on an announcement by the central bank, with respect to which the subsequent behaviour of the bank herself will result time-inconsistent. Here, if one takes the position that $\pi^{e}$ is a choice variable, there is no time inconsistency as the outcome is a Stackelberg equilibrium (i.e., the subgame perfect equilibrium of a perfect information game). If instead the idea prevails, that $\pi^{e}$ is the foresight forming in the minds of investors/consumers, then one is led to conclude that the game is affected by time inconsistency precisely because expectations are incorrect. ${ }^{7}$

The equilibrium payoffs are:

$$
L_{S}=\frac{\alpha^{3}\left(\alpha+\beta^{2}\right)(k-1)^{2} \bar{y}^{2}}{\left(\alpha^{2}+\beta^{4} \gamma\right)^{2}} ; V_{F}=\frac{\beta^{2} \gamma(k-1)^{2} \bar{y}^{2}}{\alpha^{2}+\beta^{4} \gamma}
$$

which are positive for all positive values of $k$.

\footnotetext{
${ }^{7}$ This issue also relates to the institutional arrangements that constitute the envinronment wherein inflation may more or less easily take place. Moreover, as it should be clear by now, the present analysis calls for a reassessment of the presumed causal linkage between central bank independence and low inflation (see, e.g., Posen, 1993).
} 
Finally, there remains to investigate the case where the bank takes the lead, her problem being:

$$
\begin{gathered}
\min _{\pi} L=\alpha \pi^{2}+\left[\beta\left(\pi-\pi^{e}\right)+(1-k) \bar{y}\right]^{2} \\
\text { s.t. }: \frac{\partial V}{\partial \pi^{e}}=-2\left(\pi-\pi^{e}\right)=0 .
\end{gathered}
$$

This minimum program is indeed formally equivalent to that examined in the previous section. Therefore, it entails stable prices at equilibrium, with the output at its natural level. Moreover,

$$
L_{F}=(k-1)^{2} \bar{y}^{2} ; V_{S}=0 .
$$

That is, the precommitment case is unaffected by the fact that the private sector may be inflation-averse. This is intuitive, as the case of stable prices makes the attitude of the public towards inflation completely irrelevant.

Now, one can use the expressions in (18), (23) and (25) to verify the following properties:

$$
\begin{gathered}
V_{N}>V_{F}>V_{S} \text { always; } \\
L_{N}>L_{F}, L_{N}>L_{S} \text { always; } \\
L_{F}-L_{S} \propto 1-\frac{\alpha^{3}\left(\alpha+\beta^{2}\right)}{\left(\alpha^{2}+\beta^{4} \gamma\right)^{2}}
\end{gathered}
$$

with

$$
\begin{gathered}
L_{F}-L_{S}<0 \text { for all } \gamma \in\left[0, \frac{-\alpha^{2}+\alpha \sqrt{\alpha\left(\alpha+\beta^{2}\right)}}{\beta^{4}}\right] \\
L_{F}-L_{S}>0 \text { for all } \gamma>\frac{-\alpha^{2}+\alpha \sqrt{\alpha\left(\alpha+\beta^{2}\right)}}{\beta^{4}} .
\end{gathered}
$$

The above findings can be summarised in:

Lemma 1 Suppose expectations are not necessarily correct. If so, then sequential play is always Pareto-superior to simultaneous play, and both Stackelberg equilibria are subgame-perfect equilibria of the extended game, in undominated strategies. ${ }^{8}$ However, while the private sector always prefers following to leading, the central bank prefers to lead if the public is sufficiently

\footnotetext{
${ }^{8}$ Of course, in addition to pure-strategy equilibria, there also exists a mixed-strategy equilibrium.
} 
inflation-averse, and conversely. In the Stackelberg equilibrium with the private sector leading, expectations are incorrect, while they are correct in any other outcome.

Finally, we proceed to model an alternative approach to the case where the private sector leads.

Let us assume that expectations are necessarily correct, so that $\pi^{e}=\pi$. Under this assumption, the outcome turns out to be:

$$
\pi_{F}^{e}=\frac{\beta(k-1) \bar{y}}{\alpha}=\pi_{S} ; y=\bar{y},
$$

as in (11). The equilibrium payoffs are:

$$
L_{S}=\frac{\left(\alpha+\beta^{2}\right)(k-1)^{2} \bar{y}^{2}}{\alpha} ; V_{F}=\frac{\beta^{2} \gamma(k-1)^{2} \bar{y}^{2}}{\alpha^{2}},
$$

This comes from the fact that, in this case, the public does not differentiate $V$ w.r.t. $\pi^{e}$ under the constraint (5). Rather than solving a Stackelberg leader problem w.r.t. a choice variable, the private sector simply uses $\pi^{e}$ as a pure expectation mechanism, and imposes that such expectations be correct.

If we use this solution concept, the relevant inequalities on equilibrium payoffs are qualitatively the same as in the game without aversion to inflation:

$$
L_{N}=L_{S}>L_{F} ; V_{N}=V_{F}>V_{S}
$$

and the reduced form of the extended game has the same properties of Matrix 1 , with two pure-strategy equilibria, $(S, F)$ and $(F, S)$, the latter being in weakly dominant strategies.

To sum up:

Lemma 2 Suppose expectations are correct. If so, then both Stackelberg equilibria are subgame-perfect equilibria of the extended game, but the one with the bank leading is Pareto-superior to the other and also in weakly dominant strategies. This holds irrespective of whether the private sector is inflation-averse or not.

In words, the above Lemma states that, if we drop the idea that expectations are choice variables, and just consider them as a the mechanism through which the private sector becomes fully aware in advance about the monetary 
policy selected by the central bank, then it doesn't really matter whether individuals are averse to inflation per se, or just don't care at all about it. On the contrary, in the previous setting (summarised in Lemma 1), the same consideration holds if and only if inflation is nil, in which case $\pi^{e}=\pi=0$ and $V=0$ no matter how much the public may dislike price instability.

Putting Lemmata 1-2 together, we can draw the main conclusion of our analysis:

Proposition 3 Under correct expectations, inflation may be observed as a result of randomization over the sequence according to which decisions are taken, irrespective of the attitude of the private agents toward price instability. If we allow for expectations to be incorrect, inflation obtains at a pure-strategy Stackelberg equilibrium with the private sector leading.

As a last remark, it is worth stressing that the Stackelberg equilibrium with (a) incorrect foresight and (b) the private sector taking the lead, Paretodominates all the other outcomes. Therefore, it clearly appears that under inflation-aversion the private sector does not want to correctly foresee the inflation rate produced by monetary policy. This is a typical example of a wide class of games where more information is not necessarily the better (Bassan, Scarsini and Zamir, 1997).

\section{Concluding remarks}

We have revisited the well known model of Barro and Gordon (1983a,b) by using an extended game with observable delay where the hierarchy of play is endogenously determined. In this way, we have shown that positive inflation may be observed due to mixed strategies rather than time inconsistency.

In particular, we have considered two alternative cases, according to whether private agents take care of inflation per se or not. In both cases, a positive rate of inflation can emerge, not because of the fact that central bank is unable to commit herself to the zero-inflation situation, but because the endogenous choice of the order of moves, leads to subgame perfect equilibria characterized by positive inflation.

Institutional arrangements, making clear the timing of moves, that is, the order of choices between private agents and central bank, could be effective in selecting Pareto-efficient equilibrium outcomes. 


\section{References}

[1] Alvarez, F., P.J. Kehoe and P.A. Neumeyer (2003), "The Time Consistency of Optimal Monetary and Fiscal Policies", Econometrica, forthcoming.

[2] Barro, R.J. and D.B. Gordon (1983a), "A Positive Theory of Monetary Policy in a Natural Rate Model", Journal of Political Economy, 91, 589-610.

[3] Barro, R.J. and D.B. Gordon (1983b), "Rules, Discretion and Reputation in a Model of Monetary Policy", Journal of Monetary Economics, 12, 101-21.

[4] Bassan, B., M. Scarsini and S. Zamir (1997), "I Don’t Want to Know! Can It Be Rational?" discussion paper n. 158, Center for Rationality and Interactive Decision Theory, The Hebrew University of Jerusalem.

[5] Blinder, A.S. (2000), "Central-Bank Credibility: Why Do We Care? How Do We Build It?", American Economic Review, 90, 1421-31.

[6] Calvo, G. (1978), "On the Time Consistency of Optimal Policy in a Monetary Economy", Econometrica, 46, 1411-28.

[7] Cohen, D. and P. Michel (1988), "How Should Control Theory Be Used to Calculate a Time-Consistent Government Policy?", Review of Economic Studies, 55, 263-74.

[8] d'Aspremont, C. and L.-A. Gérard-Varet (1980), "Stackelberg-Solvable Games and Pre-Play Communication", Journal of Economic Theory, 23, 201-217.

[9] Drazen, A. (2000), Political Economy in Macroeconomics, Princeton, NJ, Princeton University Press.

[10] Hamilton, J.H. and S.M. Slutsky (1990), "Endogenous Timing in Duopoly Games: Stackelberg or Cournot Equilibria", Games and Economic Behavior, 2, 29-46.

[11] Ireland, P.N. (1997), "Sustainable Monetary Policies", Journal of Economic Dynamics and Control, 22, 87-108. 
[12] Kydland, F. (1977), "Equilibrium Solutions in Dynamic DominantPlayer Models", Journal of Economic Theory, 15, 307-24.

[13] Kydland, F. and E. Prescott (1977), "Rules rather than Discretion: The Inconsistency of Optimal Plans", Journal of Political Economy, 85, 47392.

[14] Lucas, R.E. Jr. and N.L. Stokey (1983), "Fiscal and Monetary Policy in an Economy without Capital", Journal of Monetary Economics, 12, 55-93.

[15] Persson, T. and G. Tabellini (1990), Macroeconomic Policy, Credibility and Politics, Chur, Harwood Academic Publishers.

[16] Persson, T. and G. Tabellini (1999), "Political Economics and Macroeconomic Policy", in Taylor, J.B. and M. Woodford (eds), Handbook of Macroeconomics, Vol. 1c, Amsterdam, North-Holland, 1397-1482.

[17] Persson, M., T. Persson and L.E.O. Svensson (1987), "Time Consistency of Fiscal and Monetary Policy", Econometrica, 55, 1419-31.

[18] Posen, A.S. (1993), "Why Central Bank Independence Does Not Cause Low Inflation: There Is No Institutional Fix for Politics", in R. O'Brien (ed.), Finance and the International Economy: 7, Oxford, Oxford University Press, 40-65.

[19] Rogoff, K. (1987), "Reputational Constraints on Monetary Policy", Carnegie Rochester Conference Series on Public Policy, 26, 141-81.

[20] Simaan, M. and J.B. Cruz, Jr. (1973a), "On the Stackelberg Strategy in Nonzero Sum Games", Journal of Optimization Theory and Applications, 11, 533-55.

[21] Simaan, M. and J.B. Cruz, Jr. (1973b), "Additional Aspects of the Stackelberg Strategy in Nonzero Sum Games", Journal of Optimization Theory and Applications, 11, 613-26.

[22] Xie, D. (1997), "On Time Consistency: A Technical Issue in Stackelberg Differential Games", Journal of Economic Theory, 76, 412-30. 